\title{
Acute Abdomen Caused by an Infected Mesenteric Cyst in the Ascending Colon: A Case Report
}

\author{
Eun-Ji Kim, Seung-Hyun Lee, Byung-Kwon Ahn, Sung-Uhn Baek \\ Department of Surgery, Kosin University College of Medicine, Busan, Korea
}

\begin{abstract}
Mesenteric cysts are rare intra-abdominal tumors. Mesenteric cysts are usually asymptomatic and are incidentally detected during physical or radiological examination. Although uncommon, complications such as infection, bleeding, torsion, rupture and intestinal obstruction cause an acute abdomen. Spontaneous infection is a very rare complication. We present a case of infected mesenteric cysts in the ascending colon, which caused an acute abdomen. A 26-year-old woman was admitted to our hospital with acute abdominal pain. She had a painful mass in the right abdomen on physical examination. Abdominal computed tomography showed a hypodense cystic mass with septation at the mesenteric region of the ascending colon. A laparotomy revealed two cystic tumors at the mesenteric region of the ascending colon. She underwent a right hemicolectomy. The two cysts were filled with a yellowish turbid fluid. The walls of both two cysts were lined with a thin fibrotic membrane without any epithelial cell. They were diagnosed as psuedocysts with E. coli infection. Mesenferic cysts may cause life-threatening complications. Mesenteric cyst, even if it is asymptomatic and was diagnosed incidentally, should be removed completely.
\end{abstract}

Keywords: Mesenteric cyst; Infection; Abdomen, acute

\section{INTRODUCTION}

A mesenteric cyst is a cystic mass developing in the mesentery, and it is a relatively rare disease that is observed in one in 100,000250,000 hospital admissions $[1,2]$. The cause of a mesenteric cyst is still not clear; nonetheless, obstruction of lymph ducts, injury of lymph ducts, degeneration of lymph nodes, proliferation of ectopic lymphoid tissues, failure of mesenteric leaves to fuse have been considered. As its etiology, congenital diseases, diverticula, surgery in the pelvic area, and pelvic inflammatory diseases have been cited $[3,4]$. It is asymptomatic in most cases and is detected incidentally by using radiological diagnostic tests. Although uncommon, complications, such as infection, hemorrhage, volvulus, perforation,

Received: December 4, 2010 Accepted: December 23, 2010

Correspondence to: Seung-Hyun Lee, M.D.

Department of Surgery, Kosin University Gospel Hospital, 34 Amnam-dong, Seo-gu, Busan 602-702, Korea

Tel: +82-51-990-6462, Fax: +82-51-246-6093

E-mail:gscrslsh@hanmail.net

(c) 2011 The Korean Society of Coloproctology

This is an open-access article distributed under the terms of the Creative Commons Attribution NonCommercial License (http://creativecommons.org/licenses/by-nc/3.0) which permits unrestricted noncommercial use, distribution, and reproduction in any medium, provided the original work is properly cited. and ileus, have been reported [3, 5-9]. The choice of treatment is a complete surgical resection with or without bowel resection. We experienced 1 patient who developed an acute abdomen induced by infected mesenteric cysts that had developed in the mesentery of the ascending colon. The case is reported together with a review of the literature.

\section{CASE REPORT}

The patient was a 26-year-old female who was admitted for severe abdominal pain that had developed suddenly on the day of admission. Based on abdominal ultrasonography done prior to admission at another hospital, cyst torsion in the right ovary was suspected, so she was transferred to our hospital. At the time of admission, her blood pressure was $90 / 60 \mathrm{mmHg}$, her pulse was 76 times/min, her respiration rate was 24 times/min, and her temperature was $39^{\circ} \mathrm{C}$. On physical examination, tenderness, rebound tenderness, and muscle rigidity were elicited in the right abdomen. A mass approximately $10 \mathrm{~cm}$ in size was palpated in the right abdomen. On the peripheral blood test, hemoglobin was $11.7 \mathrm{~g} / \mathrm{dL}$, hematocrit was $33.9 \%$, the number of leukocytes was $12,600 / \mathrm{mm}^{3}$, and the number of platelets was $217 \times 10^{3} / \mathrm{mm}^{3}$.

On the plain abdominal X-ray, no findings of mechanical bowel 
Journal of The Korean Society of Acute Abdomen Caused by an Infected Mesenteric Cyst in the Ascending Colon

Coloproctology Eun-Ji Kim, et al.

obstruction were observed. In computed tomography, multicentric lobulated cystic mass $8 \times 5 \mathrm{~cm}$ in size was observed in the mesenteric area of the ascending colon (Fig. 1). Emergency open abdominal surgery was performed, and 2 cystic masses, $4 \mathrm{~cm}$ and $8 \mathrm{~cm}$ in diameter, in the front and the back of the mesentery of the ascending colon, respectively, were identified. Neither the appendix, nor the uterus, nor the ovaries showed special findings. A right hemicolectomy was performed. When the masses were opened, they were observed to be filled with a yellowish brown serous fluid (Fig. 2).

In histologic examination, the cysts in the mesentery were separated from the serosal layer of the ascending colon. The cyst wall was lined with a fibrous membrane with infiltration of inflammatory cells and cholesterol granules. Endothelial cells that formed the cyst wall were not detected (Fig. 3). Bacteria culture tests of the

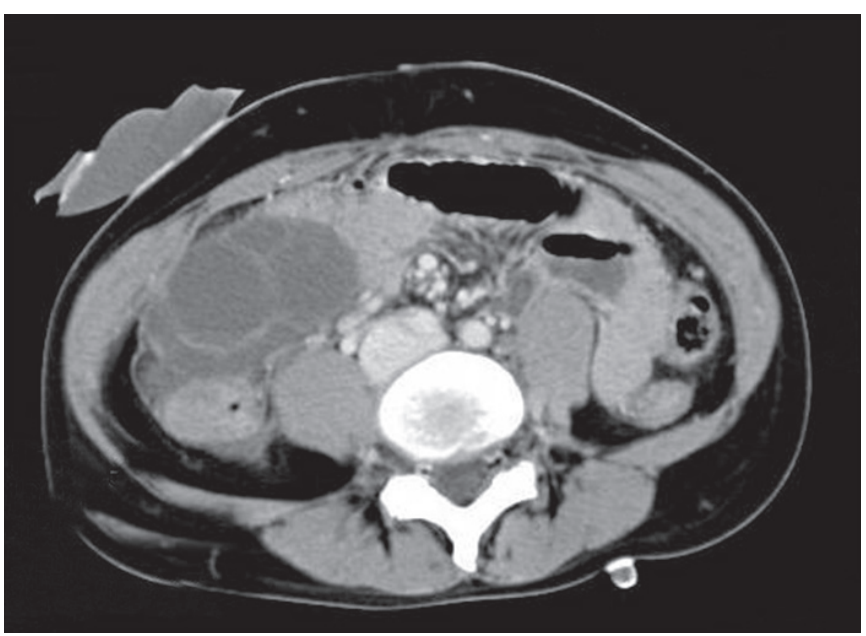

Fig. 1. Computed tomography findings for a mesenteric cyst. Multilobulated cystic mass, $8 \times 5 \mathrm{~cm}$ diameter, in the mesentery of the ascending colon.

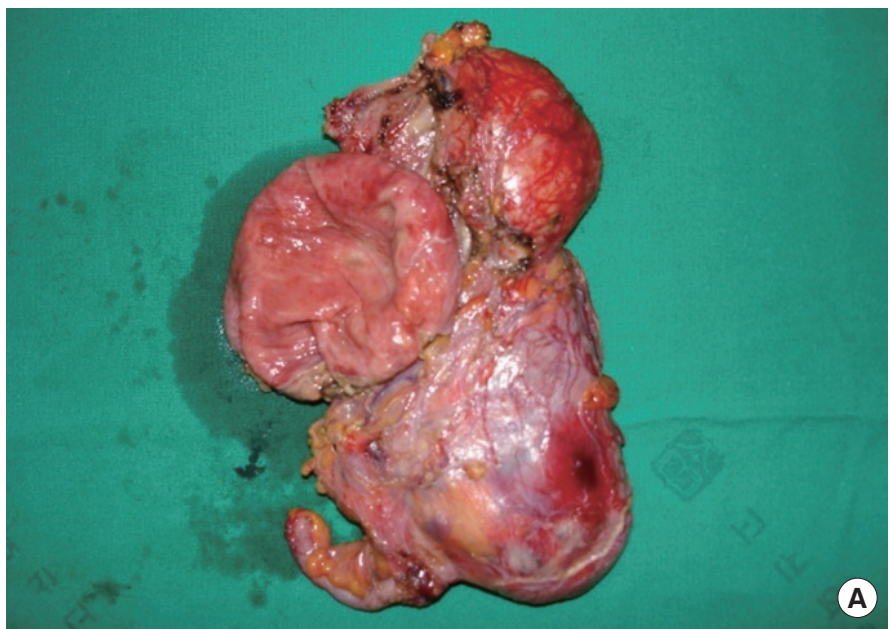

yellowish brown fluid that filled the masses were performed, and a finding of infection with Escherichia coli was shown. The patient
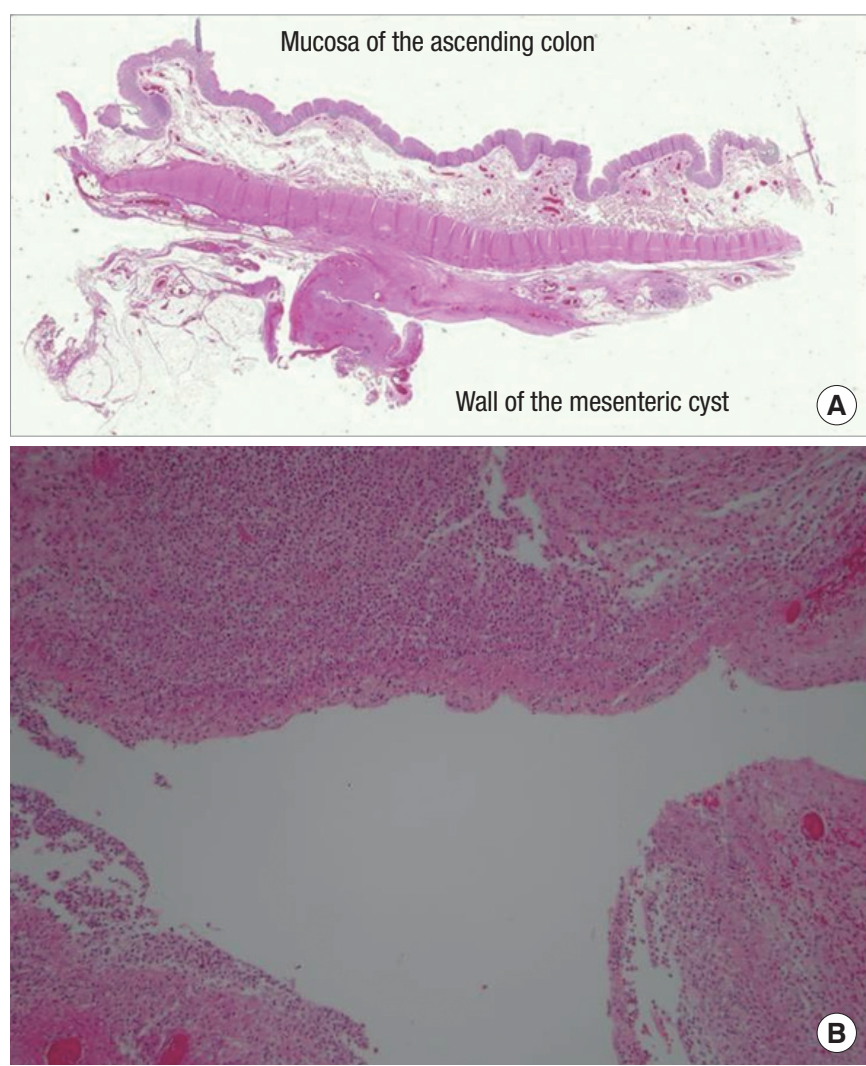

Fig. 3. Pathologic findings. (A) Wall of the mesenteric cyst is composed of a thin fibrotic membrane and is separated from the serosa of the ascending colon $(\mathrm{H} \& \mathrm{E}, \times 100)$. (B) Polymorphonuclear cells, a cholesterol granuloma, and foamy cells were scattered in the wall of the mesenteric cyst $(\mathrm{H} \& \mathrm{E}, \times 200)$.

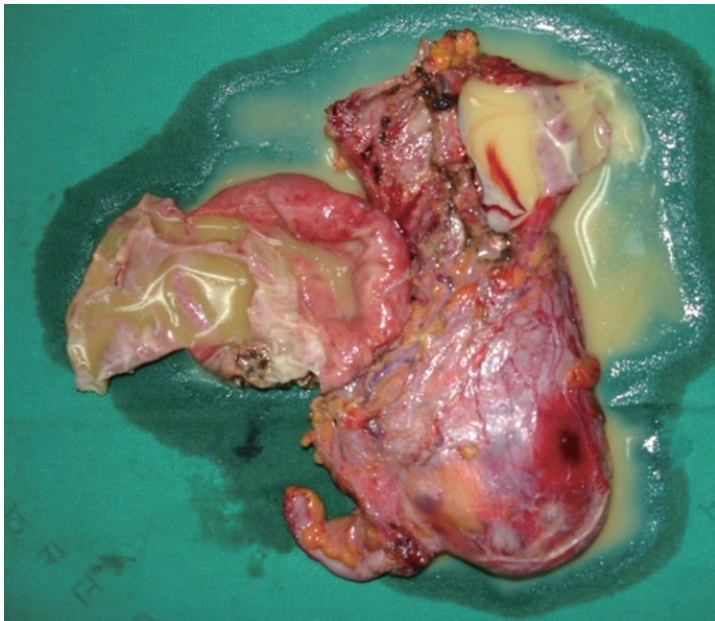

(B)

Fig. 2. Gross findings of the resected ascending colon and mesentery. (A) Two large cystic tumors $(4 \mathrm{~cm}, 8 \mathrm{~cm}$ diameter) were identified in the mesentery of the ascending colon. (B) A yellowish turbid serous fluid was collected in both cystic tumors. 
recovered without complications after surgery and was discharged on day 8 after surgery.

\section{DISCUSSION}

Mesenteric cysts have been reported to be relatively rare, occurring more frequently in females than in males and being detected in all age groups, with the 40-70 age group having a higher incidence [1, 2]. Mesenteric cysts are classified according to their etiology and histological characteristics as 1) fetal and developmental cysts, 2) traumatic or acquired cysts, 3) neoplastic cysts, and 4) infectious or degenerative cysts [10]. Among them, fetal and developmental cysts, as well as neoplastic cysts, are true cysts that are formed by endothelial cells. Traumatic cysts and infectious and degenerative cysts are classified as false cysts that are lined with a fibrous cystic wall with inflammatory cells. It has been reported that mesenteric cysts may occur in all mesenteries of the gastrointestinal tract; nevertheless, they are observed most frequently in the mesentery of the ileum $[3,9,11-13]$. Unilocular or multilocular types have been reported to be filled with serous fluid or chyle.

The sizes of mesenteric cyst are diverse. Aydinli et al. [13] reported a giant mesenteric cyst in the mesentery of the ileum whose size was $30 \times 20 \times 10 \mathrm{~cm}$, and the volume of serous fluid filling the inside was $2,500 \mathrm{~mL}$. Most cysts are asymptomatic and thus are discovered incidentally during imaging diagnostic tests or surgery. Abdominal symptoms caused by cysts, such as abdominal pain (55-82\%), palpable abdominal lumps (44-61\%), and abdominal distention (17-61\%), may be observed [3, 9, 11, 12]. Patients who develop infection, hemorrhage, volvulus, perforation, or bowl obstruction may show an acute abdomen [3, 5-9]. Shamiyeh et al. [14] stated that symptoms associated with mesenteric cysts are primarily shown in patients with cysts whose diameters are larger than 5 $\mathrm{cm}$. Sato et al. [2] reported 6 patients with mesenteric cysts; those patients showed an acute abdomen with complications primarily in young age groups. Similarly, our patient, a 26-year-old female, presented with acute abdominal pain caused by infection, with an 8 -cm maximal diameter cyst.

The inner wall of a mesenteric cyst has been reported to be composed primarily of columnar or cubic endothelial cells, but the endothelial cell layers are incomplete in some cases [15]. Cases without endothelial cells are classified as false cysts, and their causes have been reported to be trauma, infection or degeneration. In our case, the cyst wall was composed of fibrous membranes, no endothelial cells of the inner wall were detected, and infiltration of inflammatory cells and cholesterol granules was detected; thus, the case was classified as a false cyst.

False cysts frequently develop in patients in their 40s, and they are detected more in females than in males. Regarding the disease history of patients, many patients have been reported to show a disease history of abdominal trauma (30\%) and acute appendicitis (20\%), and cases diagnosed as mesenteric cysts prior to surgery are not abundant (20\%) [16]. Mesenteric cysts occur in the small in- testine, particularly in the ileum. On the other hand, the incidences of false cysts in the small intestine and the colon are comparable, and they have been reported to occur abundantly in the jejunum of the small intestine [16]. In Korea, Kim et al. [17] reported 5 cases of false cysts among 31 cases of mesenteric cysts. Min et al. [18] reported 1 case of a false cyst that developed in the ileum and induced bowel obstruction; that cyst was filled with bloody fluid. In regard to the disease history, a past history of abdominal trauma was not shown, but a past history of appendectomy was. Our case was a female in her 20 s without a past history of abdominal trauma or an appendectomy. It was a case of a false cyst that developed in the mesentery of the ascending colon. The bacteria culture test of the serous fluid that filled the inside of the cyst showed a finding of Escherichia coli infection; thus, the case was suspected to be an infectious cyst. Nonetheless, on histologic examination, no diverticula were detected in the resected ascending colon.

The choice of treatment for mesenteric cysts is complete surgical resection with or without bowel resection [19]. In our case, the cysts were located between the mesentery of the ascending colon and the retroperitoneum, and close to the ileocolic vessels and marginal vessels; thus, a simple cystectomy was difficult. Hence, surgery was performed in association with bowel resection. For cases in which the size of cyst is big and, thus, the resection area becomes broad, short bowel syndrome is anticipated, so simple aspiration or marsupialization may be considered, but generally are not recommended because of frequent recurrence and infection [9]. Mesenteric cysts are detected incidentally by using imaging diagnostic tests or during surgery in many cases, but they may cause infection, hemorrhage, volvulus, perforation, bowel obstruction and even fatal complications in some cases. Hence, when a mesenteric cyst is detected, resection should be considered.

\section{CONFLICT OF INTEREST}

No potential conflict of interest relevant to this article was reported.

\section{REFERENCES}

1. Liew SC, Glenn DC, Storey DW. Mesenteric cyst. Aust N Z J Surg 1994;64:741-4.

2. Sato M, Ishida H, Konno K, Komatsuda T, Konno S, Watanabe S, et al. Mesenteric cyst: sonographic findings. Abdom Imaging 2000; 25:306-10.

3. Sardi A, Parikh KJ, Singer JA, Minken SL. Mesenteric cysts. Am Surg 1987;53:58-60.

4. de Perrot M, Brundler M, Totsch M, Mentha G, Morel P. Mesenteric cysts. Toward less confusion? Dig Surg 2000;17:323-8.

5. Vanek VW, Phillips AK. Retroperitoneal, mesenteric, and omental cysts. Arch Surg 1984;119:838-42.

6. Alwan MH, Eid AS, Alsharif IM. Retroperitoneal and mesenteric cysts. Singapore Med J 1999;40:160-4.

7. Barut I, Tarhan OR, Ciris M, Akdeniz Y, Bulbul M. Intestinal ob- 


\section{Coloproctology Eun-Ji Kim, et al.}

struction due to a mesenteric cyst. Yonsei Med J 2004;45:356-8.

8. Ozdogan M. Acute abdomen caused by a ruptured spontaneously infected mesenteric cyst. Turk J Gastroenterol 2004;15:120-1.

9. Tan JJ, Tan KK, Chew SP. Mesenteric cysts: an institution experience over 14 years and review of literature. World J Surg 2009;33: 1961-5.

10. Beahrs OH, Judd ES Jr, Dockerty MB. Chylous cysts of the abdomen. Surg Clin North Am 1950;30:1081-96.

11. Caropreso PR. Mesenteric cysts: a review. Arch Surg 1974;108: 242-6.

12. Kurtz RJ, Heimann TM, Holt J, Beck AR. Mesenteric and retroperitoneal cysts. Ann Surg 1986;203:109-12.

13. Aydinli B, Yildirgan MI, Kantarci M, Atamanalp SS, Basoglu M, Ozturk G, et al. Giant mesenteric cyst. Dig Dis Sci 2006;51:1380-2.

14. Shamiyeh A, Rieger R, Schrenk P, Wayand W. Role of laparoscop- ic surgery in treatment of mesenteric cysts. Surg Endosc 1999;13: 937-9.

15. Bury TF, Pricolo VE. Malignant transformation of benign mesenteric cyst. Am J Gastroenterol 1994;89:2085-7.

16. Iida T, Suenaga M, Takeuchi $Y$, et al. Mesenteric pseudocyst of the sigmoid colon. J Gastroenterol 2003;38:1081-5.

17. Kim DK, Lee SO, Ryu BY, Lee YJ, Lee MC, Kim HK, et al. Mesenteric cysts: collective review of 31 cases. J Korean Surg Soc 1991; 41:458-66.

18. Min JW, Chang MC, Park YC, Go JH. Mesenteric pseudocyst causing intestinal obstruction. J Korean Surg Soc 2005;68:339-41.

19. O’Brien MF, Winter DC, Lee G, Fitzgerald EJ, O'Sullivan GC. Mesenteric cysts; series of six cases with a review of the literature. Ir J Med Sci 1999;168:233-6. 